\title{
Further discussion on the double slit experiment
}

\author{
Chong Wang* \\ College of Information Engineering, Zhejiang A\&F University, Lin'an, China
}

\begin{abstract}
In a double slit experiment, a screen position $x$ could be endowed with two probabilities: the probability of finding particles which passed through slit A and arrived in $x$, and the probability of finding particles which passed through slit B and arrived in $x$. Analysis showed that, the probability of finding particles in $x$ when two slits opened simultaneously was greater than when only one slit opened; as long as the time that the two slits opened simultaneously was long enough, the particle interference phenomenon in $x$ would occur with certainty.

Sur la base du point de vue de la classification des particules, une analyse supplmentaire est fournie: dans une exprience double fente, la probabilit de trouver une particule dans l'cran lorsque deux fentes s'ouvrent simultanment est deux fois suprieure celle d'une seule fente. Ds que le temps d'ouverture des deux fentes est suffisamment long, le phnomne d'interfrence de particules se produira certainement.
\end{abstract}

Keywords: Double slit experiment; Particle interference; Superposition state.

\section{INTRODUCTION.}

In a double slit experiment, particles exhibited wave-particle duality. Einstein presented the ensemble interpretation for the nature of particles. However, the ensemble interpretation was just a philosophical view not a critical theoretical system.

In fact, by Einstein's ensemble viewpoint and Wang's analysis[1], in a double slit experiment, a screen position $x$ could be endowed with two probabilities: the probability of finding particles which passed through slit A and arrived in $x, c_{1}$; and the probability of finding particles which passed through slit B and arrived in $x, c_{2}$.

Based on Einstein's view point, this work disclosed that, only if the time duration for the two slits that opened simultaneously was long enough, did the particle interference phenomenon always occur.

\section{ANALYSIS ON THE PROBABILITY OF FINDING PARTICLES IN THE SCREEN.}

By probability theory, the following conclusion could be obtained:

In a double slit experiment, if the probability of finding particles in $x$ when the two slits opened simultaneously was $p_{12}$, then, $p_{12}=c_{1}+c_{2}$ held. This meant that when the two slits opened simultaneously, the probability of "finding particles in $x "$ would increase.

When only one slit (A or B) opened, if the duration of the particle gun emission was $t_{1}$ seconds for slit $\mathrm{A}$ and $t_{2}$ seconds for slit $\mathrm{B}$, with $n$ particles being emitted per second, then, by probability theory, the total expected particles (for slits $\mathrm{A}$ and $\mathrm{B}$ ) in $x, N$, was:

$$
N=c_{1} n t_{1}+c_{2} n t_{2}
$$

However, when the two slits opened simultaneously, if the probability of finding particles in $x$ was $p_{12}$ and the duration of the particle gun emission was $t$ seconds, then, the expected particles in $x, N_{12}$, would be:

$$
N_{12}=p_{12} n t
$$

The condition for occurrence of the particle interference phenomenon was:

$$
N_{12}>N
$$

or

$$
p_{12} n t>c_{1} n t_{1}+c_{2} n t_{2}
$$

Which could be stated as:

$$
t>\left(c_{1} t_{1}+c_{2} t_{2}\right) / p_{12}
$$

In the laboratory, when doing a double slit experimen$\mathrm{t}$, the interference phenomenon of particles was almost certain to occur. Because in almost all experiments, $t_{1}$ and $t_{2}$ had very small values with $t$ having a large value. These would cause $t>>\left(c_{1} t_{1}+c_{2} t_{2}\right) / p_{12}$ to hold. On the other hand, when the two slits opened simultaneously, $p_{12}=c_{1}+c_{2}$ held. This meant that, when the two slits opened simultaneously, the probability of finding particles in $x$ would increase rapidly. So, in the laboratory, the particle interference phenomenon was almost certain to occur.

In practice, due to $p_{12}=c_{1}+c_{2}$ holding, even if 
$t=\left(c_{1} t_{1}+c_{2} t_{2}\right) / p_{12}$, particles were inclined to show the interference phenomenon. For example, if $c_{1}=0.45$ and $c_{2}=0.5$, then when only one slit opened, the event of "finding particles in $x$ " would be uncertain. However, when the two slits opened simultaneously, $p_{12}=0.95$ would hold meaning that the event of "finding particles in $x$ " would be almost certain. In this case, if $t=t_{1}=t_{2}=1$, then $t=\left(c_{1} t_{1}+c_{2} t_{2}\right) / p_{12}$ would hold. Thus, it could be stated that in a double slit experiment, even though $t=\left(c_{1} t_{1}+c_{2} t_{2}\right) / p_{12}$, particles tended to exhibit the interference phenomenon.

\section{DISCUSSION.}

If $\psi_{A}$ represented the wave function of particles which passed through slit A, then, $\left|\psi_{A}\right|^{2}$ should depend on $c_{1}$, $n$, and $t_{1}$. Thus, $c_{1} n t_{1}$ could be viewed as the particle probability density in $x$ as slit A opened, that is:

$$
\left|\psi_{A}\right|^{2}=c_{1} n t_{1}
$$

Likewise, if $\psi_{B}$ represented the wave function of particles which passed through slit B, then, $\left|\psi_{B}\right|^{2}$ should depend on $c_{2}, n$, and $t_{2}$. Thus, $c_{2} n t_{2}$ could be viewed as the particle probability density in $x$ as slit B opened, that is

$$
\left|\psi_{B}\right|^{2}=c_{2} n t_{2}
$$

When two slits opened, the total particle probability density in $x$ was $p_{12} n t$. Because $p_{12}=c_{1}+c_{2}$ held, if $p_{12} n t-\left(c_{1} n t_{1}+c_{2} t_{2}\right)>0$, then $\left|\psi_{A}+\psi_{B}\right|^{2}$ could be viewed as the particle probability in $x$ as two slits opened. In other words, $\left|\psi_{A}\right|^{2}$ corresponded to $c_{1} n t_{1}$, $\left|\psi_{B}\right|^{2}$ corresponded to $c_{2} n t_{2}$, and $p_{12} n t-\left(c_{1} n t_{1}+c_{2} n t_{2}\right)$ corresponded to the interference item of $\left|\psi_{A}+\psi_{B}\right|^{2}$.

Thus, when two slits opened and the interference phenomenon occurred, particles could be viewed as in the superposition state $\psi_{A}+\psi_{B}$.

\section{CONCLUSION.}

To sum up, in a double slit experiment, as long as $\delta t=t-\left(c_{1} t_{1}+c_{2} t_{2}\right) / p_{12}$ was large enough, the particle interference event was sure to happen. When the two slits opened simultaneously, the probability of "finding particles in $x "$ increased causing a tendency for particles to exhibit the interference phenomenon. When the interference phenomenon appeared, a double slit experiment would be a perfect simulation for the principle of superposition in quantum mechanics.

*390566584@qq.com

[1] Chong Wang, Phys. Essays 30, 136 (2017). 